\title{
Note
}

\section{Nonlinear Stress Relaxation of Plasticized Poly(vinyl chloride)}

\author{
Masataka Sugimoto ${ }^{\dagger}$, Takuya SuzuKi, Yuji AoKi, Sathish K. SuKumaran, and Kiyohito KoyAma \\ Graduate School of Science and Engineering, Yamagata University, \\ 4-3-16, Jonan, Yonezawa, Yamagata 992-8510, Japan
}

(Received : May 12, 2015)

\begin{abstract}
Large deformation, nonlinear stress relaxation behavior was examined for poly(vinyl chloride) in di-isononyl phthalate (PVC/DINP) system having PVC content of $45.5 \mathrm{wt} \%$ at 180,190 , and $200{ }^{\circ} \mathrm{C}$. The PVC/DINP system exhibited power law type relaxation modulus, $G(t, \gamma) \propto t^{-n^{\prime}}$, over a wide range of time $t$. The slope $n$ ' increased with elevating temperature $T$ irrespective of step-strain $\gamma$. At $190^{\circ} \mathrm{C}$, the $n$ ' value of the system was 0.75 showing a critical gel temperature $\left(T_{\text {gel }}\right)$, because 0.75 was in agreement with the values obtained from the linear dynamic viscoelasticity reported previously. Time-strain separability $G(t, \gamma)=G(t) h(\gamma)$ is applicable over a wide range of $t$. Here, $G(t)$ is linear relaxation modulus. The magnitude of damping depended strongly on $T$. Below $T_{\text {gel }}$, in other words, in a gel state, $h(\gamma)$ exhibited very weak strain dependence like cross-linking rubbers. In a critical gel state, the magnitude of damping was a little larger than that for the gel state. Above $T_{\text {gel }}$, in a sol state, $h(\gamma)$ showed still more stronger strain dependence similar to branch polymers. It was found that $h(\gamma)$ of PVC/plasticizer system strongly depended on temperature near $T_{\text {gel }}$.

Key Words: Poly(vinyl chloride) / Plasticizer / Critical gel temperature / Stress relaxation / Damping function
\end{abstract}

\section{INTRODUCTION}

It is well-known that poly(vinyl chloride) (PVC) forms gels in various solvents. The structures and physical properties of PVC gels have been extensively investigated. The gels are generally classified as randomly cross-linked networks of flexible PVC strands, and the PVC crystal domains work as the cross-linking domains. ${ }^{1,2)}$ In attempt to rheologically characterize the PVC gels, we have conducted a series of rheological studies of PVC/plasticizer systems mainly in the linear regime. ${ }^{3-14)} \mathrm{We}$ found that $\mathrm{PVC} /$ plasticizer systems exhibited a critical gel point by changing concentration ${ }^{3,7,11}$, temperature ${ }^{6,8-10,12,13)}$ and so on. ${ }^{11)}$ At the critical gel point, the dynamic storage modulus $\left(G^{\prime}\right)$ and loss modulus $\left(G^{\prime \prime}\right)$ exhibited the characteristic power-law dependence on the angular frequency w and is characterized by single exponent $n$ which is called the critical relaxation exponent that ranges between 0 and $1 .^{15,16)}$

$$
\begin{aligned}
& G^{\prime}(\omega)=G^{\prime \prime}(\omega) / \tan \delta \propto \omega^{n} \\
& \tan \delta=\tan (n \pi / 2)
\end{aligned}
$$

The loss tangent $\tan \delta$ is independent of $\omega$. Eq. 2 is of important, because this equation allows the precise value of the critical gel point. Using the relation of $G^{\prime}(\omega), G^{\prime \prime}(\omega)$ and a linear relaxation modulus $G(t)^{17)}, G(t)$ at the critical gel point can be expressed by eq. 3

$$
G(t)=S t^{-n}
$$

$S$ is called the strength of the network at critical gel point or GP-strength.

One of the nonlinear rheological measurements is uniaxial elongational test. The elongational flow behavior of polymer melts is very important for their application to polymer processing including melt spinning, foaming, bottle blowing, and so on. In a previous paper ${ }^{13)}$, we studied the dynamic viscoelasticity and elongational viscosity of PVC/di-isononyl phthalate (DINP) systems and found that the $G^{\prime}$ and $G^{\prime \prime}$ of PVC/DINP system with PVC content of 45.5 (PVC8) exhibited the power-law dependence on the angular frequency $(\omega)$ at $190{ }^{\circ} \mathrm{C}$. Correspondingly, the $\tan \delta$ value was independent of $\omega$. This temperature indicates the critical gel temperature $T_{\text {gel }}$ of the system. The critical relaxation exponent $n$ was 0.75 , which was in agreement with $n$ values reported previously by us for the low PVC concentration samples. ${ }^{3)}$ The elongational viscosity behavior of PVC8 exhibited strong strain hardening below $T_{\text {gel }}$, although it did not show any strain hardening 
above $T_{\text {gel }}$.

Another nonlinear rheological measurement is stress relaxation test. In general, nonlinear stress relaxation modulus $G(t, \gamma)$ of entangled homopolymers can be expressed by

$$
G(t, \gamma)=G(t) h(\gamma) \quad \text { for large } t
$$

where $G(t)$ is the linear relaxation modulus and $h(\gamma)$ is the damping function. ${ }^{18)} h(\gamma)$ is a universal function of $\gamma$ for entangled flexible polymers. The relaxation modulus $G(t, \gamma)$ under large deformation is another important nonlinear viscoelastic quantities. Watanabe et al. ${ }^{6}$ studied $G(t, \gamma)$ for a critical gel of PVC in di-octyl phthalate (DOP) and found that the gel exhibited nonlinear damping of the $G(t, \gamma)$ with increasing $\gamma$, but the magnitude of damping was much smaller than those for homogeneous polymer liquids. For respective materials of our interest, we will examine the time-strain separability and the damping function of critical gel systems. In this paper, we investigated the nonlinear stress relaxation modulus $G(t, \gamma)$ and damping function $h(\gamma)$ of PVC8 near the critical gel temperature $T_{\text {gel }}$.

\section{EXPERIMENTAL}

A PVC/DINP sample, coded as PVC8, was supplied from Advance Plastics Compounds, Japan. This PVC sample was synthesized via suspension polymerization and had a weightaverage degree of polymerization of 1,300 (determined from solution viscosity). The PVC powder was blended with prescribed amount of $\mathrm{Ca}-\mathrm{Zn}$ type stabilizer, and $5 \mathrm{pph}$ of $\mathrm{CaCO}_{3}$, calendered on a hot roller for $5 \mathrm{~min}$ at $150{ }^{\circ} \mathrm{C}$, compression molded at $180{ }^{\circ} \mathrm{C}$ under a pressured of $15 \mathrm{MPa}$, and cooled gradually to room temperature. The weight ratio of PVC/DINP in PVC8 was $0.455 / 0.545$. This PVC compound has a similar composition with industrial PVC/plasticizer (socalled "soft PVC") systems.

Step-shear stress relaxation measurements were carried out with a rheometer, Physica MCR-301 (Anton Paar) at 180, 190, and $200{ }^{\circ} \mathrm{C}$. Parallel-plate geometry with $8 \mathrm{~mm}$ diameter was used. The shear relaxation modulus $G(t, \gamma)$ was corrected with following equation. ${ }^{19)}$

$$
G(t, \gamma)=G_{a}(t, \gamma)\left(1+\frac{d \ln G_{a}(t, \gamma)}{4 \ln \gamma}\right)
$$

where $G_{a}(t, \gamma)$ is an apparent stress relaxation modulus measured with parallel plate. Applicability of this correction has already been demonstrated by Yoshikawa et al. ${ }^{20)}$ The time needed to impose the step strain was less than $0.1 \mathrm{~s}$. The stress relaxation behavior was measured until $100 \mathrm{~s}$, because the experimental error became larger at longer $t$. The shear strain ranged from 0.01 to 5 . All of the measurements were performed under nitrogen atmosphere. We carried out rheological measurements for the freshly prepared PVC8 system that was charged in the rheometer and heated to the experimental temperature without experiencing higher temperatures, because the interesting nonlinear features were not found for the system once heated to above the experimental temperature and then cooled to the experimental temperature.

\section{RESULTS AND DISCUSSION}

\subsection{Relaxation Modulus}

Figures 1, 2, and 3, respectively, show the nonlinear relaxation modulus $G(t, \gamma)$ of PVC8 at 180,190 , and $200{ }^{\circ} \mathrm{C}$. In these figures, the numerals denote step-strain $\gamma$. PVC8 exhibited power law type relaxations over a wide range of time

$$
G(t, \gamma) \propto t^{-n^{\prime}} h(\gamma)
$$

This implies that PVC8 has a very broad relaxation spectrum, as already explained by Osaki ${ }^{18)}$. The power-index $n$ ' of PVC8 did not vary with $\gamma$ at these temperatures. Thus, the relaxation modulus can always be separated like eq. 4 .

It was seen in Fig. 1 that the $G(t, \gamma)$ at $180{ }^{\circ} \mathrm{C}$ relaxed very slowly, exhibiting gel-like behavior. The slope $n$ ' is about $3 / 8$ irrespective of $\gamma$. It can be seen that the nonlinearity of PVC 8 was very weak and $G(t, \gamma)$ curves depended hardly on $\gamma$ despite of changing the magnitude of step strain. This suggests that PVC8 has strong network structure against large deformation at $T_{\text {gel }}$, though it is physically cross-linked gel. Figure 2 shows

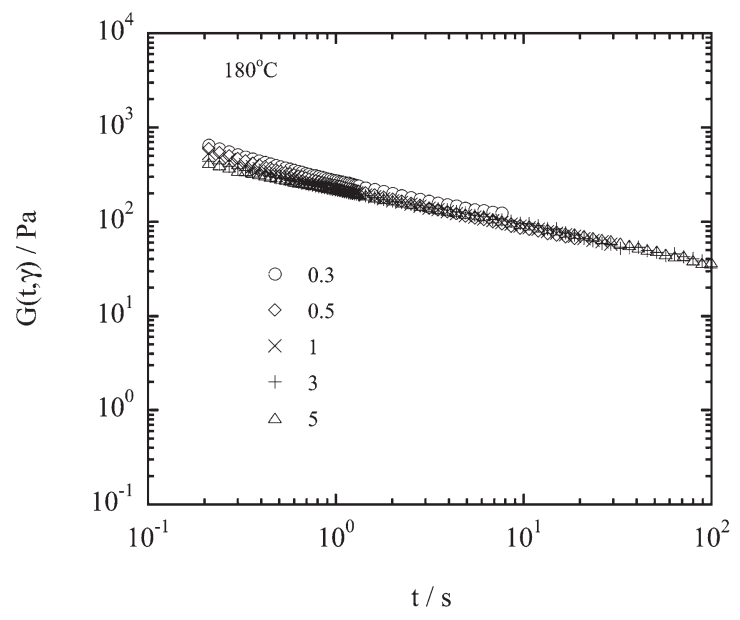

Fig. 1. Nonlinear relaxation modulus $G(t, \gamma)$ at various strain $\gamma$ for PVC8 at $180{ }^{\circ} \mathrm{C}$. 
that the $G(t, \gamma)$ at $190^{\circ} \mathrm{C}$ also relaxed very slowly. The slope, however, became steeper than that at $180{ }^{\circ} \mathrm{C}$ and the $n^{\prime}$ can be estimated to be $3 / 4$. In the previous paper $^{13)}$, we reported that the critical gel temperature $T_{\text {gel }}$ of PVC8 is $190{ }^{\circ} \mathrm{C}$ and the critical relaxation exponent $n$ is $3 / 4$ from the dynamic viscoelasticity measurements. This $n$ ' value is in good agreement with that calculated from the dynamic viscoelastic measurements by us. ${ }^{3,7,8,10-12)}$ Therefore, we should note that stress relaxation measurements also verify $T_{\text {gel }}$ of PVC8 as $190^{\circ} \mathrm{C}$. At $T_{\text {gel }}\left(=190^{\circ} \mathrm{C}\right), G(t, \gamma)$ had larger slope than that below $T_{\text {gel }}\left(180^{\circ} \mathrm{C}\right)$, and showed marginal deviation from $G(t)$, which was still small in comparison with that reported for the low PVC concentration sample ${ }^{6)}$. At $200^{\circ} \mathrm{C}$ beyond $T_{\text {gel }}, G(t, \gamma)$ relaxed more steeply, as seen in Fig. 3. The $\gamma$ dependence became larger comparing that at $T_{\text {gel }}$. This is typical behavior for flexible linear polymers.

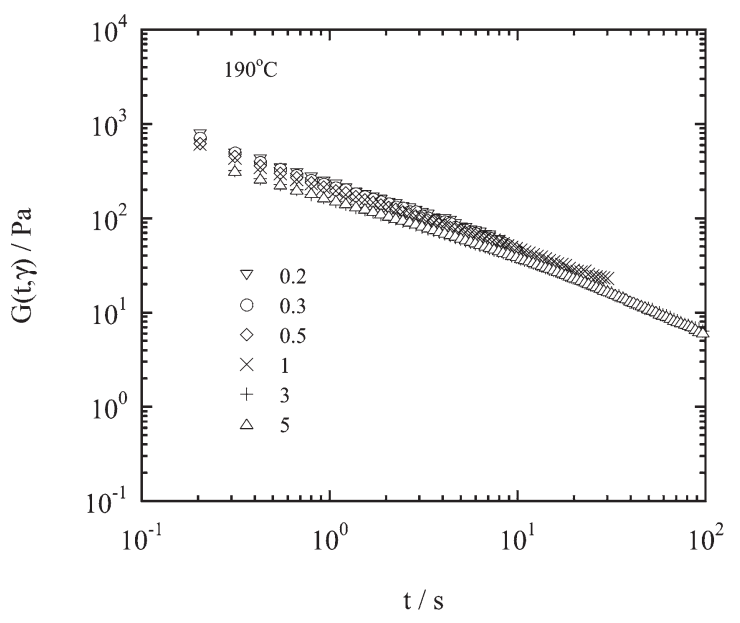

Fig. 2. Nonlinear relaxation modulus $G(t, \gamma)$ at various strain $\gamma$ for PVC8 at $190^{\circ} \mathrm{C}$.

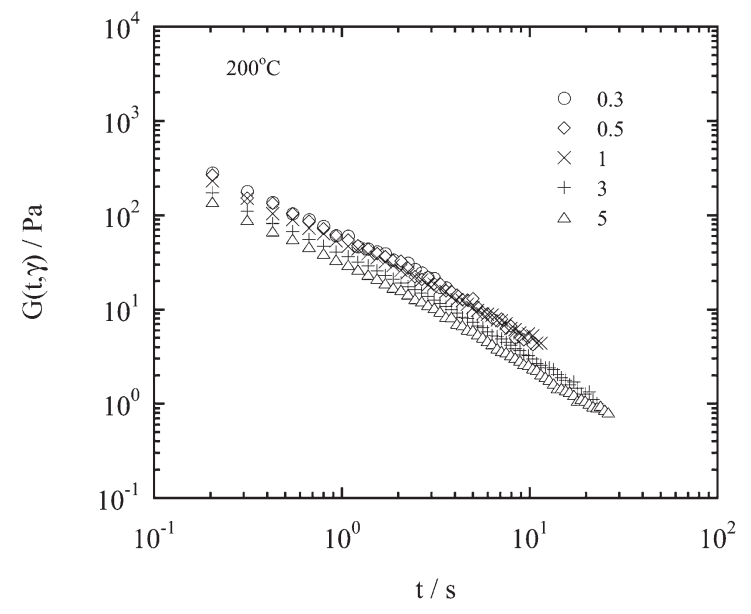

Fig. 3. Nonlinear relaxation modulus $G(t, \gamma)$ at various strain $\gamma$ for PVC8 at $200^{\circ} \mathrm{C}$.
We reported the elongational flow behavior of PVC8 at $160 \sim 190{ }^{\circ} \mathrm{C}$. Below $T_{\text {gel }}$ PVC exhibited the strong increase of elongational viscosity at large strain, which cannot be expected for linear polymers. ${ }^{13)}$ At $T_{\text {gel }}$ the strain hardening behavior was lost dramatically. This can be attributed to the change of physical cross-linked structure. The results of stepstrain experiments conducted here are also consistent with the previous studies.

\subsection{Damping Function}

The time-strain separability of PVC8 holds well over a wide range of $t$, as the slope of $\log G(t, \gamma)$ versus $\log t$ plot is the same irrespective of step-strain $\gamma$ at each temperature. Accordingly, the damping function $h(\gamma)$ can be obtained using eq. 4. Figure 4 shows the $\gamma$ dependence of $h(\gamma)$ for PVC8 at 180,190 and $200{ }^{\circ} \mathrm{C}$. For comparison, the Doi-Edward damping function ${ }^{21)}$ is shown by a dotted line. Clearly, PVC8 exhibits a much weaker $\gamma$ dependence of $h(\gamma)$ than that of Doi-Edward theory proposed for entangled linear flexible polymers. Moreover, the weaker $\gamma$ dependence of $h(\gamma)$ changes with temperature.

At $180^{\circ} \mathrm{C}$ (in a gel state), $h(\gamma)$ hardly depended on $\gamma$ like cross-linking rubbers. In the previous papers ${ }^{10,14)}$, it was reported that below $T_{\text {gel }}$ the molecular weight of the network strand between cross-links for PVC/plasticizer gels became small as decreasing temperature, in other words, cross-linking density of the gels became high. In such situation the PVC crystallites worked as the cross-linking junctions may be stable and strong in the gel state. Therefore, this physical gel exhibits no damping like cross-linked rubbers.

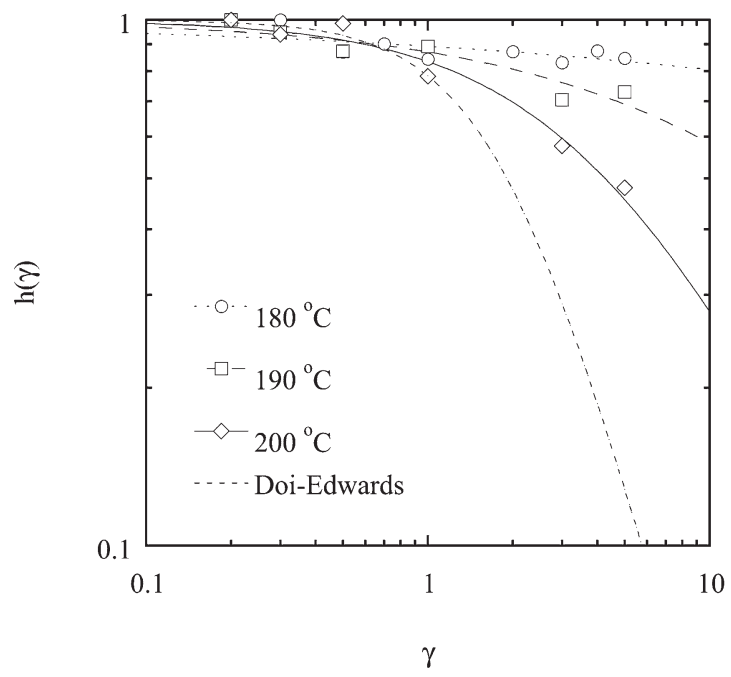

Fig. 4. Damping function $h(\gamma)$ versus strain $\gamma$ plots for PVC8 at 180 , 190 , and $200{ }^{\circ} \mathrm{C}$. 
At $190^{\circ} \mathrm{C}$ (at a critical gel), PVC8 exhibited very weak nonlinearity. Watanabe et al. ${ }^{6}$ studied nonlinear rheology for a critical PVC/DOP gel (at $49{ }^{\circ} \mathrm{C}$ ) having low PVC content $(66 \mathrm{~g} / \mathrm{L})$ and found that the magnitude of damping was much smaller than that for homogeneous polymer liquids. They discuss about the origin of weak damping function that the relaxation mechanism of the critical gel differs from the highly entangled polymer systems which can be described as the reptation dynamics. The gel strands makes it possible to sustain stress over long time scale. The shrinkage of polymeric chains occurs close to the same time as the orientation relaxation, resulting in the very weak damping. Our $h(\gamma)$ has almost the same $\gamma$ dependence with that of Watanabe et al. This fact indicates that $h(\gamma)$ has universal $\gamma$ dependence at a critical gel state irrespective of PVC concentration and plasticizer type.

At $200{ }^{\circ} \mathrm{C}$ (in a sol state), $\gamma$ dependence of $h(\gamma)$ became stronger, although PVC8 still exhibited a much weaker $\gamma$ dependence of $h(\gamma)$ than that of Doi-Edwards theory for flexible linear polymers. The temperature-dependent $h(\gamma)$ was fitted by a simple algebraic function to compare the strain softening behavior according to the following equation. ${ }^{19,22,23)}$

$$
h(\gamma)=\frac{1}{1+(a \gamma)^{2 k}}
$$

where $k$ is softening rate and $a$ corresponds to reciprocal of critical strain that the strain softening occurs. The three lines in Fig. 4 (except the line of Doi-Edwards theory) show fitted data according to eq. 7 . The rates of softening at 180 , 190 , and $200{ }^{\circ} \mathrm{C}$ were $0.15,0.34$, and 0.56 , respectively. The critical strain $\gamma_{\mathrm{c}} \sim a^{-1}$ for the onset of nonlinearity decreased with increasing temperature: $>100,17$, and 4.3 at 180, 190, and $200{ }^{\circ} \mathrm{C}$, respectively. The values of very large $\gamma_{\mathrm{c}}$ at 180 and $190{ }^{\circ} \mathrm{C}$ indicate the formation of strong network structure consisted of randomly cross-linked crystal domains and flexible polymeric strands. Uematsu et al. ${ }^{24)}$ examined nonlinear stress relaxation for $\mathrm{SiO}_{2}$ particulate gel which formed the self-similar fractal structure and were brittle. They found that the particulate gels show power-law behavior and $\gamma_{\mathrm{c}}<1$. This PVC gel, even at $200{ }^{\circ} \mathrm{C}$ (beyond $T_{\text {gel }}$ ), has still large value comparing with those of particulate gels and the linear polymers.

The important result in this paper is that the damping function of PVC/plasticizer gels showed strong temperature dependence and weak strain dependence in comparison with those of flexible entangled polymer melts. This is due to the change of fractal network structure formed by crystal domains. Watanabe et al. ${ }^{9)}$ reported that the PVC chains in the sol form fragmented (multi-branched) networks of finite sizes. Osaki ${ }^{18)}$ reported that branched polymers with two or more branch points exhibited weaker strain dependence. The weaker $\gamma$ dependence $h(\gamma)$ for PVC8 even in a sol state must reflect a multi-branched network. Thus we can say that even if the three-dimensional network disappears in PVC/plasticizer system as increasing the temperature above $T_{\text {gel }}$, PVC forms the long-chain branching chains.

\subsection{Comments on Determination of $T_{\text {gel }}$ and $n$}

Lastly, we would like to comment on determination of a critical gel temperature $T_{\text {gel }}$ and critical relaxation exponent $n$. At near $T_{\text {gel }}$ PVC8 exhibited power law type $G(t, \gamma)$. This power law behavior is characterized by a self-similar fractal structure. The slope $n$ ' changed from about $3 / 8$ at $180{ }^{\circ} \mathrm{C}$ to above 1 at $200{ }^{\circ} \mathrm{C}$ through $3 / 4$ at $190{ }^{\circ} \mathrm{C}\left(T_{\text {gel }}\right)$. Above findings implies that the gel consists of different self-similar structures near $T_{\text {gel }}$. However, we should note that it is not appropriate to determine from stress relaxation experiments, since the form of eq. 3 may contain contradictions to the experimental observations. ${ }^{6,15)}$ In the step-strain experiments, the polymer is subjected to very high strain rate until the desired strain. In general, when the time during the step-strain is enough short comparing with the relaxation time, eq. 3 for the transient shear modulus is valid. In our case eq. 3 is adequate. However, we need to note reproducibility of the power-law behavior of $G(t)$. Since PVC gel with self-similarity has broad distribution of cross-linking mesh size, the short strands connecting small crystal domains worked as cross-linking points may be affected by rapid deformation during the step-strain motion as mentioned by Watanabe et al. ${ }^{6}$ Although we confirmed for our high polymer concentration system that the power-law behavior of the fresh sample (without deformation history) was coincident with the once-strained sample, the relation has not yet been elucidated through the experimental method.

The slope $n$ ' is very important physical constant for gelling systems. Therefore, for accurate determination of $T_{\text {gel }}$, we must make plots of $\tan \delta$ against temperature for a set of angular frequency from the dynamic viscoelastic measurements. ${ }^{8,13,14)}$ Then, we can obtain precise $T_{\text {gel }}$ and $n$.

\section{CONCLUDING REMARKS}

We have reported the nonlinear stress relaxation behavior of PVC/DINP system. The system exhibited power law type relaxation modulus, $G(t, \gamma) \propto t^{-n^{\prime}}$, over a wide range of $t$. The slope $n$ ' increased with elevating temperature irrespective of $\gamma$. At $190{ }^{\circ} \mathrm{C}$, the $n$ ' value of the system was 0.75 showing a 
critical gel temperature $\left(T_{\text {gel }}\right)$, because the critical gel exponent $n=0.75$ at $T_{\text {gel }}$ was in agreement with the result of dynamic viscoelasticity. The time-strain separability, $G(t, \gamma)=G(t)$ $h(\gamma)$, is applicable. The magnitude of damping depended on temperature. Below $T_{\text {gel }}, h(\gamma)$ exhibited very weak strain dependence like cross-linking rubbers. In a critical gel state, the magnitude of damping was a little larger than that for the gel state. Above $T_{\text {gel }}, h(\gamma)$ showed strain dependence like branch polymers. Our results would contribute to develop new thermoplastic elastomers using PVC/plasticizer systems.

\section{REFERENCES}

1) Guenet GM, "Thermoreversible Gelation of Polymers and Biopolymers", (1992), Academic Press, London.

2) Te Nijenhuis K, Adv Polym Sci, 130, 1 (1997).

3) Li L, Aoki Y, Macromolecules, 30, 7835 (1997).

4) Li L, Uchida H, Aoki Y, Yao M-L, Macromolecules, 30, 7842 (1997).

5) Li L, Aoki Y, Macromolecules, 31, 740 (1998).

6) Watanabe H, Sato T, Osaki K, Aoki Y, Li L, Kakiuchi M, Yao M-L, Macromolecules, 31, 4198 (1998).

7) Aoki Y, Li L, Kakiuchi M, Uchida H, Kakiuchi M, Watanabe H, Macromolecules, 31, 7472 (1998).

8) Aoki Y, Li L, Kakiuchi M, Macromolecules, 31, 8117 (1998).
9) Watanabe H, Osaki K, Kakiuchi M, Aoki Y, Macromolecules, 34, 666 (2001).

10) Kakiuchi M, Aoki Y, Watanabe H, Osaki K, Macromolecules, 34, 2987 (2001).

11) Kakiuchi M, Aoki Y, Watanabe H, Osaki K, Nihon Reoroji Gakkaichi (J Soc Rheol Jpn), 29, 53 (2001).

12) Aoki Y, Macromolecules, 34, 3500 (2001).

13) Sugimoto M, Hida H, Taniguchi $T$, Koyama K, Aoki $Y$, Rheol Acta, 46, 957 (2007)

14) Aoki Y, Hirayama K, Kikuchi K, Sugimoto M, Koyama K, Rheol Acta, 49, 1071 (2010).

15) Winter HH, Chambon F, J Rheol, 30, 367 (1986).

16) Chambon F, Winter HH, J Rheol, 31, 683 (1987).

17) Ferry JD, "Viscoelastic Properties of Polymers", $3^{\text {rd }}$ ed, (1980), John Wiley \& Sons Inc, NY.

18) Osaki K, Rheol Acta, 32, 429 (1993).

19) Soskey PR, Winter HH, J Rheol, 28, 625 (1984).

20) Yoshikawa T, Toneaki N, Moreki Y, Takahashi M, Masuda T, Nihon Reoroji Gakkaishi (J Soc Rheol Jpn), 18, 80 (1990).

21) Doi M, Edwards SF, "The Theory of Polymer Dynamics", (1986), Clarendon, Oxford.

22) Islam MT, Sanchez-Reyes J, Archer LA, J Rheol, 45, 61 (2001).

23) Ng TSK, McKinley GH, J Rheol, 52, 417 (2008).

24) Uematsu H, Aoki $Y$, Sugimoto M, Koyama K, Rheol Acta, 49, 1187 (2010). 